\title{
Nosocomial infections and infectious risks related to hospital environment
}

\author{
Nabila Saouide El Ayne ${ }^{1,2}$, Hinde Hami ${ }^{2}$, Samir Hamama ${ }^{3}$, Sanou Khô Coulibaly ${ }^{1}$, Abdelrhani Mokhtari ${ }^{2}$, and Abdelmajid \\ Soulaymani ${ }^{2}$
}

${ }^{1}$ Faculty of Medicine and Odonto-Stomatology, University of Sciences, Techniques and Technologies, Bamako, Mali

${ }^{2}$ Laboratory of Biology and Health, Faculty of Science, Ibn Tofail University, Kenitra, Morocco

${ }^{3}$ Quality unit, El Idrissi Hospital, Kenitra, Morocco

\begin{abstract}
Introduction: The fight against nosocomial infections, particularly those linked to environmental germs, is one of the priorities of health establishments. Microbiological control of the hospital environment is an essential element of the strategy for the fight against these infections. The aim of this study is to determine the microbial ecology of hospital surfaces and medical devices. Methods: This is a study, which was carried out in the El Idrissi hospital in Kénitra. For the microbiological control of surfaces, we opted for the swab technique: the search for specific germs on flat surfaces and in areas that are difficult to access and not flat. Results: Among the bacteria most frequently responsible for isolated nosocomial infections: Bacillus (28\%) and coagulase-negative staphylococci $(27 \%)$ were predominant, followed by Staphylococcus aureus (19\%), Klebsiella pneumoniae (17\%), Pseudomonas aeruginosa (5\%), Enterobacter cloacae (3\%) and Proteus vulgaris (1\%).The overall distribution of germs by department showed their predominance in intensive care units $(30 \%)$, traumatology $(11 \%)$, emergencies $(11 \%)$ and in operating rooms $(9 \%)$. Conclusion: Periodic microbiological controls must be put in place in order to identify any contamination, to carry out preventive actions, procedures and a fundamental approach for the control of infectious risks in the hospital.
\end{abstract}

Keywords: Nosocomial infections; Hospital environment; Morocco

\footnotetext{
*Corresponding author: nabila.saouide@gmail.com
} 


\section{Introduction}

Nosocomial infections represent a real public health problem in the world because they cause an increase in mortality, morbidity and an extra cost related especially to the increasing the length of stay. Their prevalence rate, in the world, varies between $1 \%$ and $20 \%$ and the overall incidence from $5 \%$ to $10 \%$ with a variation from country to another [1].

According to the World Health Organization (WHO), nosocomial infections affect 5 to $10 \%$ of patients admitted to modern hospitals in developed countries [2]. In countries developing, these infections affect $25 \%$ or more of patients [2].

Microbiological monitoring of the hospital environment is one of the pillars of the policy to fight nosocomial infections. According to several studies, microorganisms responsible for these infections were Staphylococcus aureus, Klebsiella pneumoniae, Escherichia coli and Pseudomonas aeruginosa [3, 4]. The departments most affected by these infections were the surgical, intensive care, burn unit, medicine, pediatrics, obstetric gynecology, and trauma department $[5,6]$.

The factors responsible for nosocomial infections are the patients (age and immune status), medical practices and hospital environment [7]. The term hospital environment usually includes surfaces, water, air, laundry, food, medical devices and waste. We will limit ourselves in this article to surfaces and medical devices (operating table, respirators, cart, bed.....).

The aim of this study is to conduct a microbiological surveillance of hospital surfaces of medical devices for each department of the hospital for the evaluation of the percentage of contamination of these and the identification of isolated germs.

\section{MATERIALS AND METHODS}

\subsection{Site of study}

A prospective study was carried out over a period of seven months at the El Idrissi hospital in Kenitra with a total bedding capacity of 418 beds. The sampling of surfaces and medical devices has been conducted in all departments of the hospital.

\subsection{Sampling techniques}

According to ISO / DIS 14698-1, we performed the swab technique in both specific cases: the search for specific germs in the area flat (tables of operations, operating lights, material trolleys, night tables, beds, floor ...) and areas that are difficult to access and not level (medical devices, wrists, humidifier, breathing pipes, bubblers, liquid respirators, sterilizers, incubators, stethoscopes, cupboards ...).

Sterile swabs pre- moistened in sterile isotonic solution. At each site selected area was swabbed with parallel streaks, closely spaced and repeated in two directions at right angles by performing a tight zigzag while rotating the swab. Sampling of each site was repeated three times in order to obtain representative results. Afterwards, the swabs were put back in their protective cases and were sent to the laboratory within a quarter of an hour. Then the swabs were moistened in tubes containing brain-heart infusion broth (BHI Brain Heart Infusion Broth), then incubated for 24 to 48 hours at $37{ }^{\circ} \mathrm{C}$. In total, we took 210 samples.

\subsection{Sample analysis}

In isolation, we used several culture media: Bromcresol Purple Lactose Agar, middle Chapman, blood and chocolate agar. The Petri dishes were incubated at $37^{\circ} \mathrm{C}$ for 24 to 48 hours, the abundance of colonies is noted and Gram-negative bacilli colonies were transplanted on a gallery classic and API 20E, then identified using standard keys and Bergey's manual of systematic bacteriology 1984. Each sample was repeated and identified 3 times.

\subsection{Data processing and analysis}

The data entry was carried out using Microsoft Office Excel 2013. The descriptive and analytical parts were carried out using the XLSTAT extension.

The $\mathrm{p}$-value $<0.05$ was considered significant.

\section{RESULTS}

Among the 210 samples taken, 195 were found to be positive, ie a frequency of contamination of $93 \%$ (Figure 1).

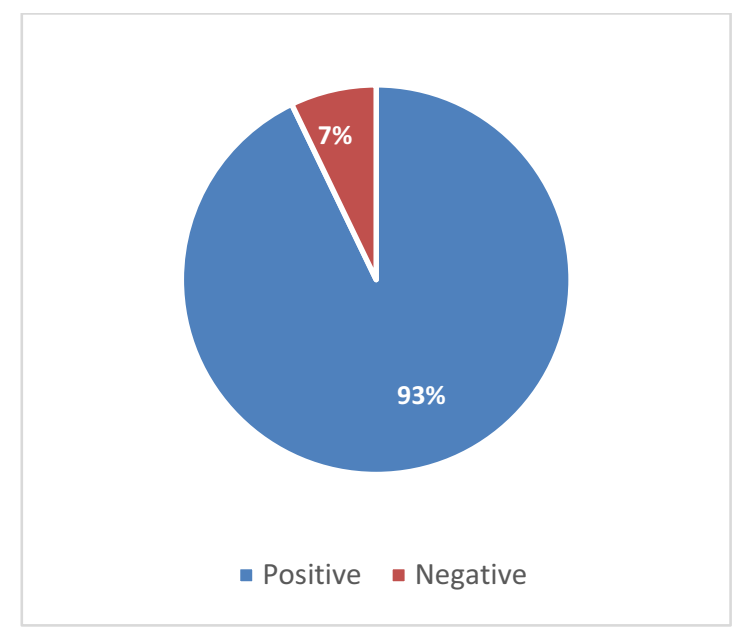

Fig. 1. Contamination frequency of surfaces

The overall distribution of germs by department showed their predominance in the intensive care units $(30 \%)$, traumatology $(11 \%)$, emergencies $(11 \%)$ and operating rooms $(9 \%)$ (Figure 2$)$. 


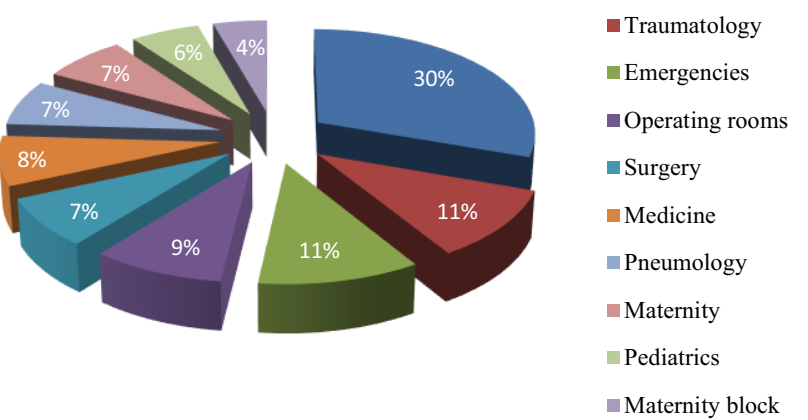

Fig. 2. Global distribution of germs by service

In total, the most frequently isolated germs: Bacillus (28\%) and coagulase-negative staphylococci $(27 \%)$ were predominant, followed by Staphylococcus aureus (19\%) and Klebsiella pneumoniae (17\%). Finally, Pseudomonas aeruginosa in a proportion of 5\%, Enterobacter cloacae (3\%) and Proteus vulgaris in 1\% (Figure 3).

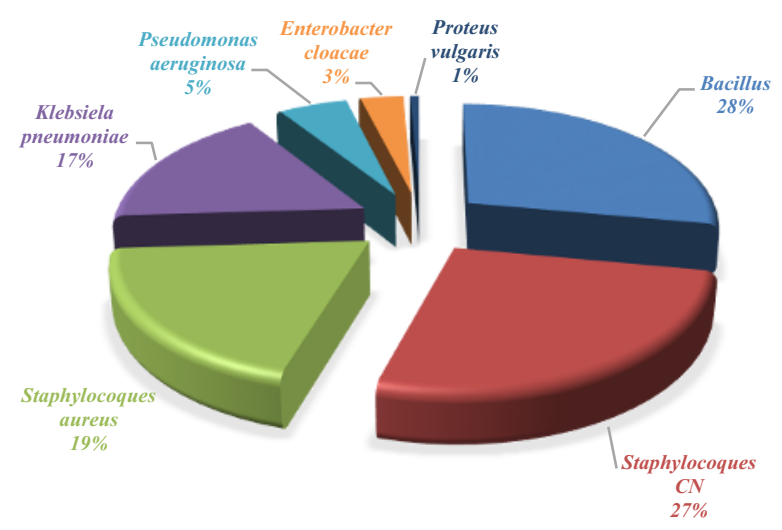

Fig. 3. Distribution of germs isolated from hospital surfaces

Among the germs, Bacillus were omnipresent in all departments with a predominance in the operating rooms as well as coagulase-negative staphylococci in surgery, medicine, pneumology and maternity. Klebsiella pneumoniae were isolated in all departments except the maternity ward with a proportion of $34 \%$ in the pediatrics. As well as Staphylococcus aureus were isolated in almost all hospital wards. Pseudomonas aeruginosa was isolated only in intensive care units by $16 \%$ and Proteus vulgaris by $2 \%$ (Figure 4 ).

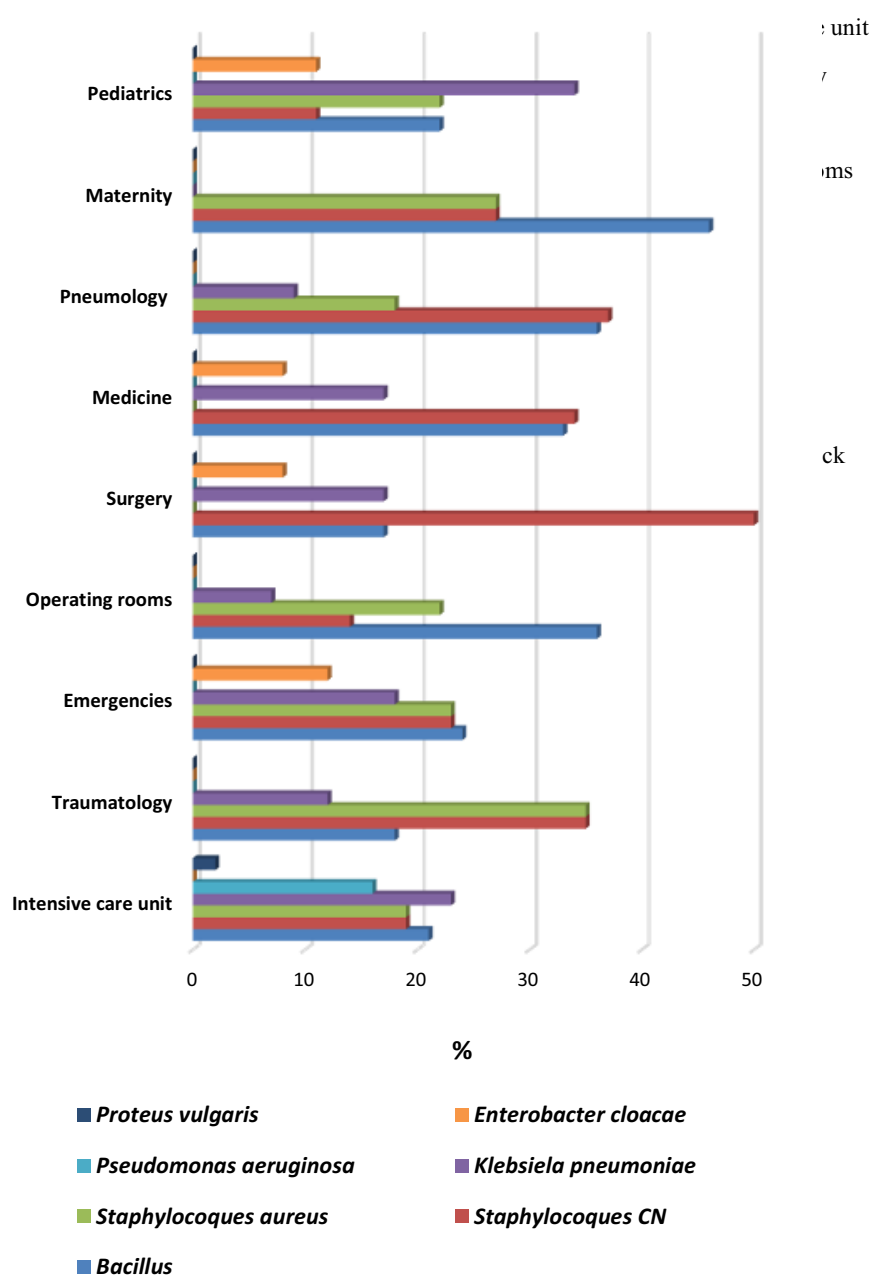

Fig. 4. Distribution of germs in hospital services

\section{DISCUSSION}

The hospital environment is usually contaminated with microorganisms manmade and environmental $[8,9]$. This contamination of this environment varies qualitatively and quantitatively over time, from one hospital or establishment to another and, within the same establishment, depending on the services, patients, care and practiced techniques. According to studies, It has been shown that the immediate environment of a patient carrier bacteria multi - resistant antibiotic (BMR) can be contaminated by himself and subsequently act as a secondary tank [10].

A study carried out in a university hospital with 1,500 beds evaluated the environmental contamination of these 35 operating theaters over a period of 9 months [11]. On 283 samples made, $16.6 \%$ were positive for bacterial pathogens (Bacilles to Gram negative, Staphylococcus aureus, Enterococcus sp.), $57.6 \%$ were positive for bacteria of the skin flora. The soil was the site most often 
found contaminated in this study $(65 \%$ of samples were positive).

Munoz Price et al. (2012) have also observed that objects fall to the ground are frequently replaced on the operating table. Also that the IV tubes are frequently in contact with the ground, consequently the anesthetists and nurses have multiple contacts with these tubes as well as the patients, which can promote the transmission of microorganisms [11].

Another study has shown that phone block procedures are contamined on average with 23.3 CFU per telephone, most of the isolated germs: coagulase-negative staphylococci (82.8\%), Acinetobacter baumannii (1.9\%) and Pseudomonas aeruginosa (1.9\%) [12].

The contamination of surfaces does not depend only on the quality of the bio-cleaning but on many factors linked to microorganisms: Its lifespan on an inert support, the latter varies according to the material, the temperature and the desiccation. Also depends on its adhesion to the surface, its ability to produce a biofilm and its ability to resist unfavorable conditions (sporulation). In this sense, it has been shown that Staphylococcus aureus and Acinetobacter baumannii are the species among the most resistant to desiccation and can survive for several weeks on dry surfaces. Some Enterobacteriaceae can survive for more than a week [13, 14]. Clostridium difficile may persist, in sporulated form, for several months [15].

In our case, Staphylococcus aureus took third place by $20 \%$ after coagulase-negative staphylococci. In addition, they were isolated almost in all the hospital services, which testifies to a general deficiency of the hospital hygiene. On the other hand, Pseudomonas aeruginosa were isolated only in re- animation (19\%).

Escherichia coli, Enterobacterium, the most common in nosocomial infections is much less resistant to desiccation [16-14]. In our case, Enterobacter cloacae were isolated in a few departments such as emergencies (15\%) and pediatrics (16\%). Particularly long survival times, reaching more than 6 months are described, in particular with certain epidemic strains of Staphylococcus aureus [17]. Under moist conditions and in the presence of organic matter, survival is even longer [16].

Our study showed that $93 \%$ of the samples were found positive. Resuscitation was the most affected service with $30 \%$ contamination, followed by traumatology $(11 \%)$ and emergency $(10 \%)$. Thereafter, operating rooms, surgery, medicine and pneumology by $8 \%$. Finally, maternity by $6 \%$, pediatrics by $7 \%$ and maternity block by $6 \%$.

Contamination of the operating room, trauma and surgery has also been demonstrated [18]. Also, that the contamination of the operating room [19].

Meunier et al. (2005) have found that devices and surfaces near patients are heavily contaminated with pathogenic microorganisms [20]. The results of our study showed that hospital surfaces and devices medical the hospital are contaminated with pathogens that can cause nosocomial infections.

Our study showed a $93 \%$ contamination rate. This rate is comparable to that found by other studies. Berrada et al. (2017) reported a positivity rate of $96 \%$ before the installation of the hemodialysis center at Al Ghassani hospital in Fès (Morocco) and of 76.2\% after renovation of the center [21]. Meunier et al. (2005) reported a positivity rate of $87 \%$ in Strasbourg [22]. Also, another study revealed a contamination rate of $97.92 \%$ in Fès [23]. However, Meité et al. (2010) showed a lower percentage of contamination of $46.7 \%$ in Abidjan (Ivory Coast) [24].

\section{CONCLUSION}

Nosocomial infections are responsible for prolonging the length of stay with increased economic cost and are associated with significant mortality.

The results of this study have shown that hospital surfaces are contaminated with pathogenic germs which represent a significant cause of nosocomial infections. It should also be noted that there are indirect arguments which most often allow the environment to be implicated as a primary or potentially secondary tank at the origin of the infection. This study shows the importance of prevention, which remains the only way to limit the risk of nosocomial infection, based on the establishment of a disinfection protocol applied to reusable medical surfaces and devices. Also, the hospital hygiene training is an essential element in the prevention of nosocomial infections. It must be individualized in a specific way in each establishment because it contains all the clinical, microbiological and epidemiological aspects of these infections. It must be offered to all departments and to all personnel, as an essential element of continuing training and it is an indicator of quality and safety.

\section{References}

1. WHO, WHO Guidelines on Hand Hygiene in Health Care: A Summary. First Global Patient Safety Challenge Clean Care Is Safer Care (2019)

2. WHO, Pourquoi un Défi Mondial Sur les Infections Nosocomiales (2018)

3. K. Amazian, J. Rossello, A. Castella, S. Sekkat, S. Terzaki, L. Dhidah, Eastern Mediterranean Health Journal, 16, 1070-1078 (2010)

4. K. EL Rhazi, S. Elfakir, M. Berraho, N. Tachfouti, Z. Serhier, C. Kanjaa, C. Nejjari, Eastern Mediterranean Health Journal, 13, 56-63 (2007)

5. M. Khouchoua, Enquête de Prévalence des Infections Associées aux Soins au Centre Hospitalier Régional Mohamed V Meknès (Thesis, 2013)

6. A. Chaibdraa, M.C. Bentakouk, Ann. Burns Fire Disasters, 21, 7-12 (2008)

7. Ministère de la Santé Marocaine, Manuel d'Hygiène Hospitalière et de Prévention des Infections Nosocomiales (2008)

8. W.A. Rutala, D.J. Weber, Infect. Control Hosp. Epidemiol., 18, 609-616 (1997)

9. D.J. Weber, W.A. Rutala, Environmental issues and nosocomial infections, in R.P. Wenzel, Prevention and control of nosocomial infections, 3rd Ed, Williams \&Wilkins, 491-514 (1997)

10. D. Talon, J. Hosp. Infect., 43, 13-17 (1999)

11. L.S. Munoz-Price, D.J. Birnbach, D.A. Lubarsky, K.L. Arheart, Y. Fajardo-Aquino, M. Rosalsky, T. 
Cleary, D. DePascale, G. Coro, N. Namias, P. Carling, Infect. Control Hosp. Epidemiol., 33, 897904 (2012)

12. J. Nelson, A. Bivens, A. Shinn, L. Wanzer, C. Kasper, AORN J., 83, 607-611 (2006)

13. S. Oie, A. Kamiya, J Hosp Infect, 34, 145-149 (1996)

14. C. Wendt, B. Wiesenthal, E. Dietz, H. Ruden, J. Clin. Microbiol., 36, 3734-3736 (1998)

15. R. Barbut, S. Getty, D. Neyme, S. Magne, Y. Bernardon, R. Ribadeau Dumas, J.C. Petit, Hygienes, 6, 449-455 (2003)

16. A. Jawad, J. Heritage, A.M. Snelling, D.M. Gascoyne-Binzi, P.M. Hawkey, J. Clin. Microbiol., 34, 2881-2887 (1996)

17. J.H.T. Wagenvoort, W. Sluijsmans, R.J.R. Penders, J. Hosp. Infect., 45, 231-234 (2000)

18. A. El Ouali Lalami, H. Touijer, F. El-Akhal, M. Ettayebi, N. Benchemsi, S. Maniar, H. Bekkari, Journal of Materials and Environmental Science, 7, 123-130 (2016)

19. S. Jaouhar, A. El Ouali Lalami, K. Ouarrak, J. Bouzid, M. Maoulouaa, K. Bekhti, Scientifica, 1318480 (2020)

20. O. Meunier, C. Hernandez, M. Piroird, R. Heilig, D. Steinbach, A. Freyd, Ann. Biol. Clin., 63, 481-486 (2005)

21. S. Berrada, G.B. Touimi, L. Bennani, A.S. Diarra, B. Oumokhtar, A. El Ouali Lalami, F.Z. Squali Houssaini, T. Sqalli Houssaini, Revue Francophone Internationale de Recherche Infirmière, 3, 120-128 (2017)

22. O. Meunier, C. Hernandez, M. Piroird, R. Heilig, D. Steinbach, A. Freyd, Ann. Biol. Clin., 63, 481-486 (2005)

23. H. Touijer, Prévention des infections nosocomiales : écologie bactérienne en milieu hospitalier et sensibilité aux anti- biotiques et aux désinfectants (Thesis, 2014).

24. S. Méité, C. Boni-Cissé, P. Monemo, A.P. Mlantanoa, H. Faye-ketté, H. Dosso, J. Sci. Pharm. Biol., 11, 73-81 (2010) 